\title{
A hand-guided robotic drill for cochleostomy on human cadavers
}

This article was published in the following Dove Press journal:

Robotic Surgery: Research and Reviews

\author{
Xinli Du' \\ Peter N Brett ${ }^{2}$ \\ Yu Zhang' \\ Philip Begg ${ }^{3}$ \\ Alistair Mitchell-Innes ${ }^{3}$ \\ Chris Coulson ${ }^{3}$ \\ Richard Irving ${ }^{3}$ \\ 'Brunel Institute for Bioengineering, \\ Brunel University London, Uxbridge, \\ UK; ${ }^{2}$ University of Southern \\ Queensland, Toowoomba, QLD, \\ Australia; ${ }^{3}$ University Hospitals \\ Birmingham NHS Foundation Trust, \\ Birmingham, UK
}

Correspondence: Xinli Du Brunel Institute for Bioengineering, Brunel University London, Kingston Ln, Uxbridge UB8 3PH, UK

Email xinli.du@brunel.ac.uk
Background: An arm supported robotic drill has been recently demonstrated for preparing cochleostomies in a pilot research clinical trial. In this paper, a hand-guided robotic drill is presented and tested on human cadaver trials.

Methods: The innovative smart tactile approach can automatically detect drilling mediums and decided when to stop drilling to prevent penetrating the endosteum. The smart sensing scheme has been implemented in a concept of a hand guided robotic drill.

Results: Experiments were carried out on two adult cadaveric human bodies for verifying the drilling process and successfully finished cochleostomy on three cochlea. The advantage over a system supported by a mechanical arm includes the flexibility in adjusting the trajectory to initiate cutting without slipping. Using the same concept as a conventional drilling device, the user will also be benefit from the lower setup time and cost, and lower training overhead.

Conclusion: The hand-guided robotic drill was recently developed for testing on human cadavers. The robotic drill successfully prepared cochleostomies in all three cases.

Keywords: surgical robot, hand guided robot, smart sensing, drilling cochleostomies, hearing preservation, cochlear implantation

\section{Introduction}

Over the last 30 years, robotic surgery has made its mark as a precise mean of tool deployment in surgical procedures. ${ }^{1,2}$ It has demonstrated consistent results ${ }^{3-5}$ for certain procedures, such as laparoscopic surgery, with reduced length of stay and blood loss. ${ }^{6,7}$ For many other procedures, the upfront cost, consumable costs, surgeon training overhead, and maintenance of a large system cannot be justified. ${ }^{8}$ At the meanwhile, a number of hand-guided robotic systems, which are smaller and intuitive to use, have been developed, for example, assisting gripping tissues (laparoscopy), guiding hand-held instruments, and cutting applications (knee joint replacement surgery). ${ }^{8-12}$ Hand-held robots have the advantage of being compact and easily integrated into routine surgical practice. These devices have a physically smaller footprint, make use of much of the surgeon's existing dexterity, and are typically lower in cost with minimal setup time and lower training overhead. ${ }^{13}$ The development of such devices faces the crucial challenge of achieving successful results within a less-structured working environment such as deforming tissue, and they need the robustness to accomplish this with disturbances both induced by surgeon and patient motions. Sensing systems, protocol, and configuration need to address this challenge. 
An innovative tactile method to automatically discriminate mediums and structures ahead on a cutting tool trajectory has been demonstrated successfully in surgery to produce precise cochleostomies. ${ }^{14}$ The method enables preservation of fine tissue structures by simultaneous determining of the state of the process and automatically stopping the drilling if undesired drilling medium is detected. Most importantly, this is used to achieve high tissue preservation and low tissue trauma in surgery. ${ }^{15-17}$ This tactile tissue-guided sensing approach enables extension of an arm-supported robotic drill explored as a hand-guided unit. It relies on an innovative method for tactile sensing to determine and respond to the state of both the tissue being drilled and the tissue about to be drilled.

A cochlear implant is a surgically implanted device that allows rehabilitation of hearing in patients with severeto-profound hearing loss. It represents the gold standard treatment for patients who derive limited or no benefit from conventional hearing aids. The anatomy of ear is shown in Figure 1 with indication of the position of cochleostomy. The cochlear implant is inserted inside cochlear through the cochleostomy hole.

Residual hearing preservation has attracted increasing attention in recent years. Poor preservation of tissue could cause poor hearing preservation during the implantation process. Although it is an ongoing debate about the optimal procedure for opening cochlear through cochleostomy or round window, sometimes cochleostomies cannot be avoided if the round window is difficult to access. Among different stages in the surgical procedure of cochlear implantation, cochleostomy is considered crucial to hearing preservation. The reasons are twofold, the considerable chance of inadvertent perforation being the first. Inadvertent perforation is destructive as it exposes the cochlea to perilymph contami-

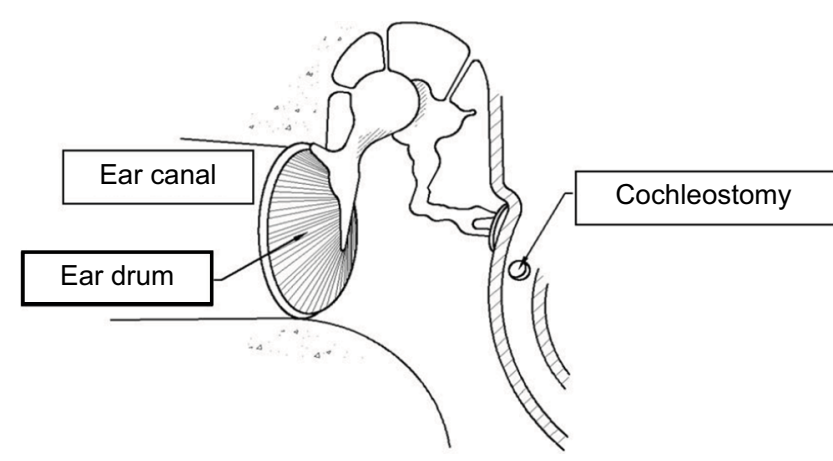

Figure I Diagram illustrating the anatomy of the ear and location of a cochleostomy. Note: Reproduced with the permission of John Wiley and Sons. Du X, Assadi MZ, Jowitt F, et al. Robustness analysis of a smart surgical drill for cochleostomy. Int J Med Robot. 2013;9(1):119-126.21 nation - by bone dust and exotic fluid such as blood, and the risk of drill bit entering scala vestibuli and potentially damaging the basilar membrane where sensory cells are located. Second, the action of drilling on the delicate central sensing organ can cause acoustic mechanical trauma - inner ear trauma resulted from excessive acoustic stimuli or in general mechanical disturbance. Drill-induced mechanical trauma is proven to be severe in middle ear surgery especially if the ossicular chain is drilled unintentionally. Using a robotic device to perform cochleostomy could help to improve the consistency and accuracy. Several robotic devices have been developed for minimally invasive cochlear implantation. ${ }^{18-20}$ Such robotic devices require high-resolution computed tomography (CT) images for the operator to preplan the drilling path ${ }^{18,19}$ or calibrate the robot. ${ }^{20}$ During the surgery, image navigation system is used to track the movement of the robotic arm relative to the patient. Primarily, such robotic device development is focused on creating access tunnel to cochlea avoiding facial nerve during the drilling process. In contrast, the present research is focusing on the opening of cochlear. The presented robotic device is in the format of a hand-guided device for easy setup and handling. The device does not require preplanned trajectory and works similar to the conventional drill. The advantage is that it can automatically decide when to stop the drilling before entering undesired layer of the structure, ie, endosteum. The unique smart sensing algorithm uses information of the interaction between the tool and the drilling medium to discriminate the drilling stage. In this article, a human cadaver trial for a hand-guided robotic drill is presented to evaluate the setup and performance of the device in a clinical environment.

\section{Methods}

\section{Hand-guided robotic drill}

The concept of a hand-guided robotic drill has been inspired by an automated, mechanical arm-supported, robotic drill recently applied in clinical practice to produce cochleostomies. ${ }^{17}$ The smart sensing algorithm uses information derived from coupled force and torque transient discriminating tissue boundaries/structures ahead on the drilling path. This valuable approach robustly detects and preserves the endosteum underlying bone tissue of the cochlear capsule to produce a membrane window of correct diameter ready for electrode insertion into the cochlea. The process achieves precise feed characteristics with micron-level accuracy to deform tissue boundaries. Earlier successful clinical trials demonstrated reduced disturbances in tissues, thus reducing trauma to the inner ear. Novel methods of measurement 
indicate that the technique reduces peak-to-peak amplitude of intracochlear disturbances to $1 \%$ of manual drilling. ${ }^{17}$

A hand-held drill is more convenient to use than a device constrained by a mechanical support arm. From the perspective of surgeons, who are used to deploy tools by hand, it is likely to appear more intuitive to use. Previous research has proved that the flexibility in the drilling trajectory will help the control of drilling into the basal turn of the cochlea. Initial cutting without slip is achieved more readily when the drilling trajectory is perpendicular to the surface..$^{20,21}$

The hand-guided drilling system contains three units, such as a drill unit, a hard-wired control unit, and an output screen. Figure 2 shows the system containing all the three units, and Figure 3 shows the drill unit. The drill unit uses standard drill bit driven by a servo motor. The design of the chuck helps to change the drill bit easily and transfer the pushing force to the sensor inside the unit. The hardwired control unit contains two microcontrollers. One is to provide servo control of the drill unit, and the other is to control the information communication to the output screen through ethernet. There are also LED bars on the control unit showing the pushing force during drilling. It is important to maintain the pushing force in the range between 0.5 and

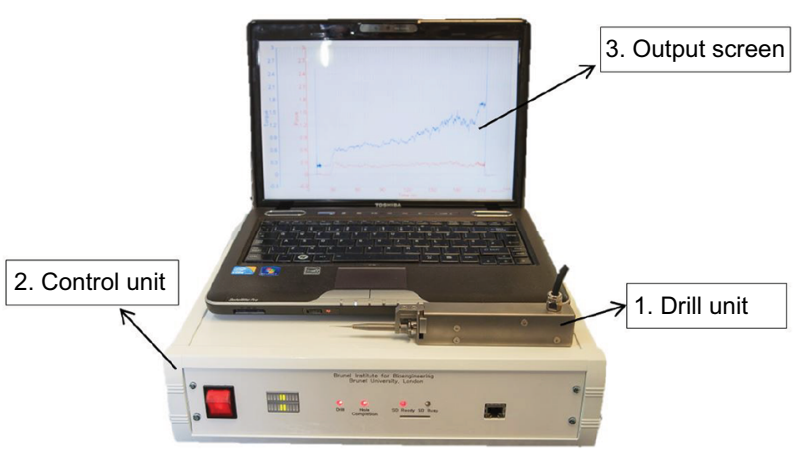

Figure 2 The experimental hand-guided surgical robot drill system.

Note: Open Access Creative Commons, Brett P, Du X, Zoka-Assadi M, Coulson C, Reid A, Proops D. Feasibility study of a hand guided robotic drill for cochleostomy. Biomed Res Int. 2014;2014:7.22
$1.5 \mathrm{~N}$ shown as green area on the LED bars. If pushing too hard or too light, the LED bar will display red. On the output screen, a user interface is displayed to show information such as pushing force, rotation torque, and rotation speed. The system has been tested on a variety of phantoms such as raw eggs and porcine cochlear. ${ }^{21}$ The feasibility results demonstrated the consistency and robustness when drilling on variety phantoms.

\section{Cadaver experiments}

The cadaver trials were carried out on two adult cadaveric human bodies bequeathed for medical education and research purposes. Specimens were obtained within $120 \mathrm{~h}$ of death and frozen at $-20^{\circ} \mathrm{C}$. Experiments were carried out within 3 months of death. Otoscopy and tympanometry were carried out prior to temporal bone drilling. To achieve easy access to the promontory and the basal turn of the cochlea, a wide cortical mastoidectomy and posterior tympanotomy were performed on each side of the head of each specimen. Care was taken to retain the ear canal wall intact throughout the whole experimental procedure to make sure that middle ear transfer function can be measured at different stages. The ossicular chain and the inner ear were examined carefully, and no abnormality was found. Although the purpose of experiments was not to investigate middle ear mechanism, the tympanic membrane, ossicular chain, and all ligaments and tendons were preserved throughout the whole experimental process. This was to eliminate any effect of an incomplete sound conducting system on the cochlear dynamics.

The drilling was performed by an ENT surgeon for both preparing the access to cochlea and then drilling the cochleostomy. Written informed consent was provided by the person in Figure 4 to have the image published. The drilling process is shown in Figure 4. A total of $1 \mathrm{~mm}$ diameter diamond burrs were used during the trials. The robotic drill was held by the surgeon's left hand resting on the armed chair to avoid
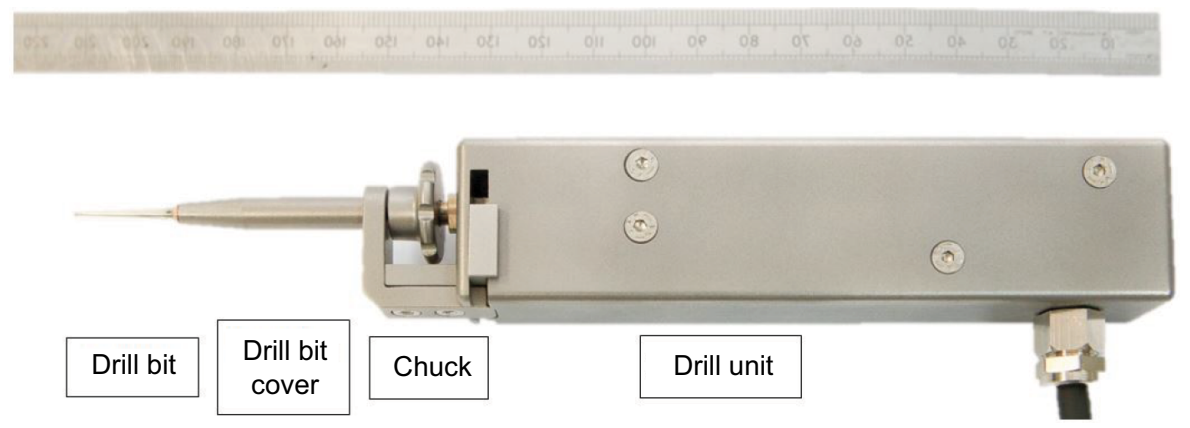

Figure 3 The hand-guided robotic drill unit.

Note: Open Access Creative Commons, Brett P, Du X, Zoka-Assadi M, Coulson C, Reid A, Proops D. Feasibility study of a hand guided robotic drill for cochleostomy. Biomed Res Int. 2014;2014:7.22 
too much movement. In theater, one would use a shoulder bolster next to the patients' head to allow direct wrist support and minimization of tremor. ${ }^{23,24}$ The drilling processes were performed under a surgical microscope. The drill bit was first applied to the desired position before the drilling process started. The drill process started by pressing the start button on the control user interface. After starting, the operating surgeon guided the drill unit forward to perform the drilling. Pressure applied throughout the robotic drilling process was monitored and kept constant - the surgeon was able to correct the force applied according to a real-time signal. The drill process will automatically stop when a cochleostomy was created before penetrating the endosteum. The drilling can be stopped by the operator at any time of the drilling process for checking or cleaning if needed. The operator can continue drilling by following the same process of start drilling to finish the cochleostomy.

\section{Ethic approval}

This work was approved by University Research Ethics Committee of the Brunel University London with a reference number 3129-TISS-Jun/2016- 3192-1.

\section{Results}

Four cases of cochleostomies were performed. The first cochlea was primarily used for the surgeon to practice the use of robotic drill on - mitigating the gap in surgeon's experience with using conventional drilling. The other three cases of cochleostomy were successfully finished with intact underlying endosteal membrane on two cadaver heads. One finished result of a cochleostomy is shown in Figure 5. The underlying membrane remained successfully intact.

The correlated coupled force and torque transients are shown in Figure 6. The force level during drilling was maintained at $\sim 1 \mathrm{~N}$ over the range from 0.6 to $1.3 \mathrm{~N}$. The operator begins by increasing the feed force to ensure that the drill is cutting and is stable on the surface. The result is an initial force building transient. Following this period, the fluctuating force amplitude is primarily due to unsteady motion imparted by the operator. This could be due to the unusual posture to support the drill, which is in need of improvement as simply indicated in the earlier section. At the end of the drilling process (56 s), a rapid increase in the torque and dropping of the force can be observed. These coupled characteristics together are indicative of completion of the cochleostomy. Although significant disturbances induced by operator's hand tremor and movement are present in the signals, the automated discrimination of completion of the cochleostomy is not interrupted and the robotic drilling process successfully completes the cochleostomy as required.

\section{Discussion}

In this article, hand-guided robotic drilling is shown to be a beneficial process over that of conventional drilling for avoiding inadvertent penetration of the delicate endosteum. The sensing technique enables control of drilling to produce accurate and consistent results relative to tissue interfaces. The robot is in the same form as conventional surgical drills,

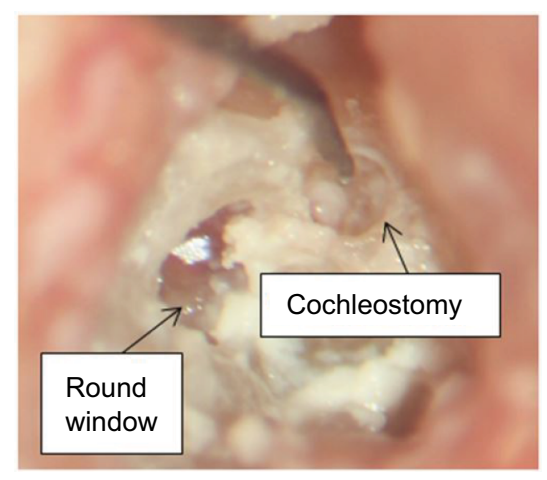

Figure 5 The finished cochleostomy using both hand-guided robotic drill and conventional surgical drill with endosteal membrane.

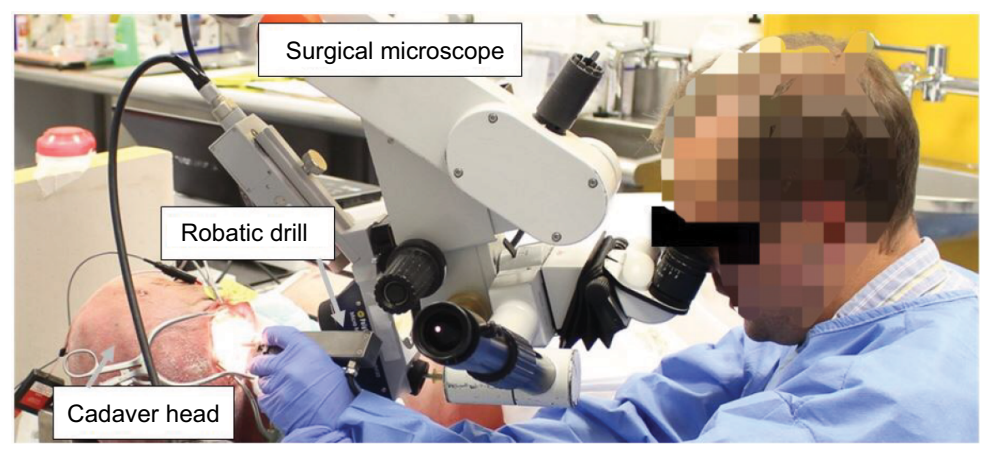

Figure 4 Drilling cochleostomy using hand-guided robotic drill on cadaver. 


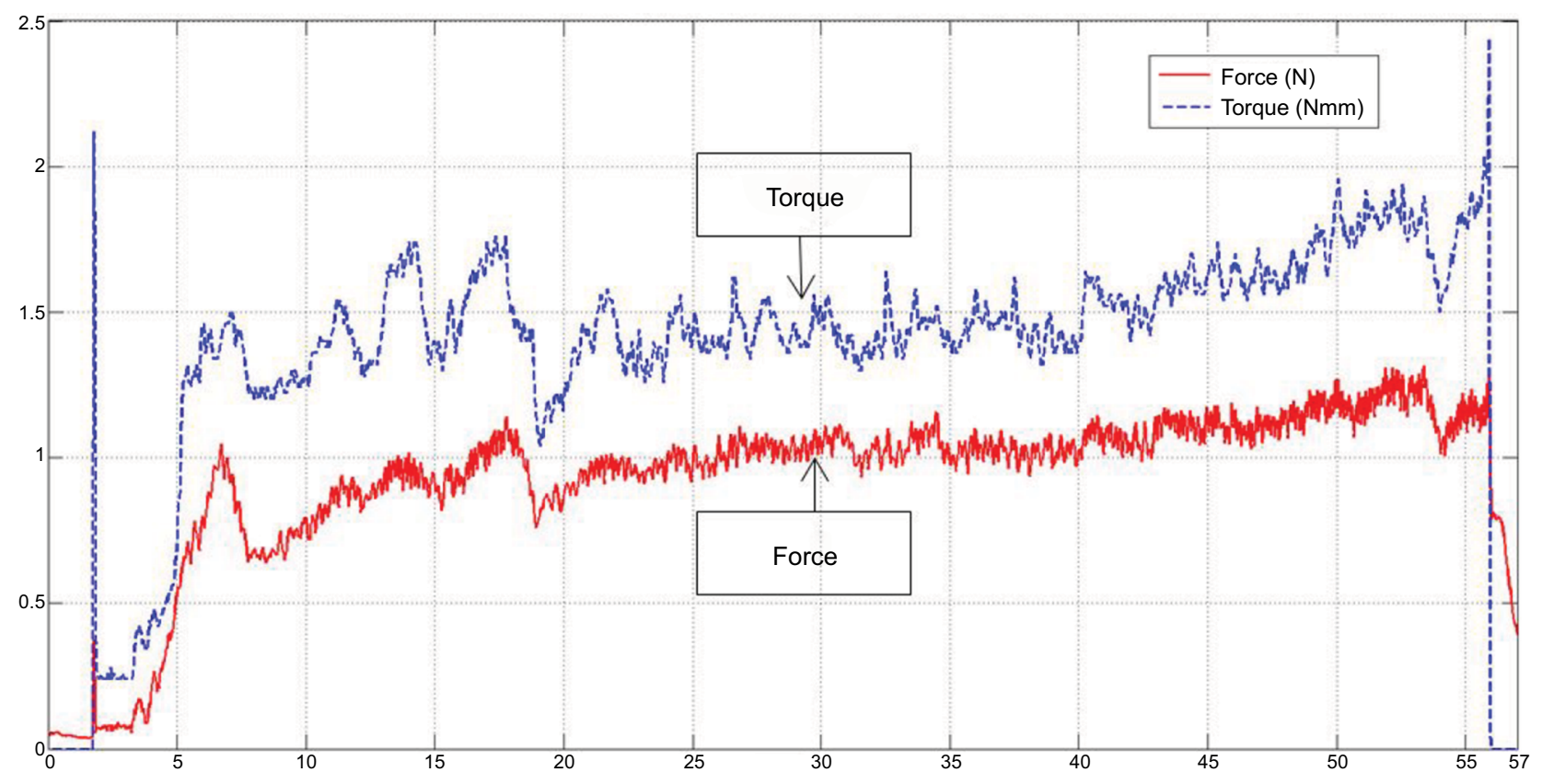

Figure 6 A typical force and torque transient during the drilling.

such that it can be easily integrated into existing surgical procedures without any significant training time. The robotic device is also similar in the size and setting up time compared to conventional surgical drill. The familiar form of the device, weight, and balance, with that of a conventional drill, enables ready application by the operator. Compared to other robotic systems for cochlear implantation discussed in the studies by Caversaccio et al, ${ }^{18}$ Majdani et al, ${ }^{19}$ and Nguyen et al,,${ }^{20}$ the presented robotic drill does not require high-resolution CT scan information or preplanned trajectory. The robotic drill is hand guided by the operating surgeon so that no further optical tracking system is needed. Instead of simply following the preplanned path, the robotic drill can automatically discriminate the drilling stage and make decision when to stop drilling before the penetration of endosteum. At the meanwhile, the system can feedback and record the drilling information, ie, applied force and the rotating torque, to the operator for monitoring purpose.

The presented system is focused on the procedure for opening cochlea during the cochlear implantation. It is an ongoing debate regarding the best route for cochlear insertion, whether directly through the round window or after the creation of a cochleostomy. However, an accurately placed cochleostomy may provide a better insertion angle compared to a round window approach, as shown in Meshik et al, ${ }^{25}$ subsequently reducing the likelihood of trauma later in the insertion process. Although it does not provide the function for creating a minimally invasive tunnel to cochlea, the unique sensing technology can be integrated into a robot for such purpose, as discussed in Williamson et al. ${ }^{26}$ The combination of the smart drill with the robotic system could enable a more fully integrated minimally invasive procedure spanning minimally invasive access through the facial recess and atraumatic cochleostomy.

The former mechanical arm-supported version of the robotic drill has been used in the operating room and has shown significant reduction in intracochlear disturbances induced while drilling a cochleostomy. ${ }^{17}$ There is anticipated benefit in the reduction of tissue trauma as a result. Further investigation will be required to contrast the reduction in disturbances induced using the robotic hand-guided drilling solution over that of conventional devices, such that the beneficial contribution toward reducing trauma is known.

\section{Conclusion}

The robotic microdrilling method applied to surgery can discern tissue interfaces ahead on a drill path. This enables tools to cut up to delicate tissue interfaces without penetration automatically. Applied to a cochlear implantation procedure, the process can be deployed to advantageously maximize tissue preservation and minimize trauma during surgery. This article presented the trial for creating cochleostomy on two human cadaver heads using the hand-guided drill. This helps to verify the performance of the device in a clinical setup and environment. The endosteum was remained induct in three cases of drilling while one cochlea was used for training 
the surgeon to operate the robotic device. The hand-guided robotic drill produces consistent outcomes and augments surgeon control and skill. The advantage over an arm-supported system is that it offers flexibility in adjusting the trajectory. This can be important to initiate cutting without slipping and then to proceed on the desired trajectory.

\section{Disclosure}

The authors report no conflicts of interest in this work.

\section{References}

1. Drake JM, Joy M, Goldenberg A, Kreindler D. Computer and robotic assisted resection of brain tumours. Proceedings of the Fifth International Conference on Advanced Robotics. Pisa: ICAR; 1991:888-892.

2. Taylor RH, Paul HA, Mittelstadt BD, et al. An image based robotic system for hip replacement surgery. J Robot Soc Jpn. 1990;8(5): 111-116.

3. Guthart GS, Salisbury JK. The intuitive telesurgery system: overview and application. IEEE International Conference on Robotics and Automation (ICRA '00). San Francisco, California. Vol. 1. 2000:618-621.

4. Jakopec M, Rodriguez y Baena F, Harris SJ, et al. The hands-on orthopaedic robot 'acrobot': early clinical trials of total knee replacement surgery. IEEE Trans Robot Autom. 2003;19(5):902-911.

5. Lonner JH, John TK, Conditt MA. Robotic arm-assisted UKA improves tibial component alignment: a pilot study. Clin Orthop Relat Res. 2010;468(1):141-146.

6. Hu JC, Gu X, Lipsitz SR, et al. Comparative effectiveness of minimally invasive vs open radical prostatectomy. JAMA. 2009;302(14):1557-1564.

7. Ramsay C, Pickard R, Robertson C, et al. Systematic review and economic modelling of the relative clinical benefit and cost-effectiveness of laparoscopic surgery and robotic surgery for removal of the prostate in men with localised prostate cancer. Health Technol Assess. 2012;16(41):1-313.

8. Laskaris J, Regan K. Soft Tissue Robotics - The Next Generation (Vol. VII). MD Buyline (2014).

9. Jess HL, Glenn JK. Robotically assisted unicompartmental knee arthroplasty. Oper Tech Orthop. 2012;22(4):182-188.

10. Jaramaz A, Nikou C, Simone A. NavioPFS for unicondylar knee replacement: early cadaver validation. Bone Jt J. 2013;95-B(Suppl 28):73.
11. Schuller B, Rigoll G, Can S, Feussner H. Emotion sensitive speech control for human-robot interaction in minimal invasive surgery. $R O-M A N$ 2008. The 17th IEEE International Symposium on Robot and Human Interactive Communication. Munich, Germany. Vol. 458. 2008:453.

12. Nelson CA, Zhang X, Shah BC, Goede MR, Oleynikov D. Multipurpose surgical robot as a laparoscope assistant. Surg Endosc. 2010;24(7):1528-1532.

13. Payne CJ, Yang GZ. Hand-held medical robots. Ann Biomed Eng. 2014;42(8):1594-1605.

14. Taylor RP, Du X, Proops DW, Reid AP, Coulson C, Brett PN. A sensoryguided surgical micro-drill. Proc Inst Mech Eng C J Mech Eng Sci. 2010;224(7):1531-1537.

15. James C, Albegger K, Battmer R, et al. Preservation of residual hearing with cochlear implantation: how and why. Acta Otolaryngol. 2005;125(5): 481-491.

16. Zou J, Bretlau P, Pyykkö I, Starck J, Toppila E. Sensorineural hearing loss after vibration: an animal model for evaluating prevention and treatment of inner ear hearing loss. Acta Otolaryngol. 2001;121(2):143-148.

17. Coulson CJ, Zoka Assadi M, Taylor RP, et al. A smart micro-drill for cochleostomy formation: a comparison of cochlear disturbances with manual drilling and a human trial. Cochlear Implants Int. 2013; 14(2):98-106.

18. Caversaccio M, Gavaghan K, Wimmer W, et al. Robotic cochlear implantation: surgical procedure and first clinical experience. Acta Otolaryngol. 2017;137(4):447-454.

19. Majdani O, Rau TS, Baron S, et al. A robot-guided minimally invasive approach for cochlear implant surgery: preliminary results of a temporal bone study. Int J Comput Assist Radiol Surg. 2009;4(5):475-486.

20. Nguyen Y, Miroir M, Vellin JF, et al. Minimally invasive computerassisted approach for cochlear implantation: a human temporal bone study. Surg Innov. 2011;18(3):259-267.

21. Du X, Assadi MZ, Jowitt F, et al. Robustness analysis of a smart surgical drill for cochleostomy. Int J Med Robot. 2013;9(1):119-126.

22. Brett P, Du X, Zoka-Assadi M, Coulson C, Reid A, Proops D. Feasibility study of a hand guided robotic drill for cochleostomy. Biomed Res Int. 2014;2014:7.

23. Hildmann H, Sudhoff H. Middle Ear Surgery. Springer Science \& Business Media; 2006. Berlin, Germany.

24. Coulson CJ, Slack PS, Ma X. The effect on wrist rest on hand tremor. Microsurgery. 2010;30(7):565-568.

25. Meshik X, Holden TA, Chole RA, Hullar TE. Optimal cochlear implant insertion vectors. Otol Neurotol. 2010;31(1):58.

26. Williamson T, Du X, Bell B, et al. Mechatronic feasibility of minimally invasive, atraumatic cochleostomy. Biomed Res Int. 2014;2014: 181624.
Robotic Surgery: Research and Reviews

\section{Publish your work in this journal}

Robotic Surgery: Research and Reviews is an international, peer reviewed, open access, online journal publishing original research, commentaries, reports, and reviews on the theory, use and application of robotics in surgical interventions. Articles on the use of supervisory-controlled robotic systems, telesurgical devices, and shared-control systems are

\section{Dovepress}

invited. The manuscript management system is completely online and includes a very quick and fair peer review system, which is all easy to use. Visit http://www.dovepress.com/testimonials.php to read real quotes from published authors. 